\title{
Correlation Functions of a Lattice System
}

\author{
G. Gallavotti and S. Miracle-Sole \\ Institut des Hautes Etudes Scientifiques, Bures-sur-Yvette
}

Received November 23, 1967

\begin{abstract}
We deduce an integral equation for the infinite volume correlation functions of a class of lattice systems and we apply it to find results on the analyticity in the interaction potentials of the pressure and of the correlation functions and on the ergodicity of the equilibrium states in the gaseous phase. By similar methods we prove some cluster properties for the correlation functions in the gaseous phase.
\end{abstract}

\section{$\S 1$. Introduction and Notations}

In this paper we study the infinite volume correlation functions of a class of classical systems of particles on a $\nu$-dimensional lattice $Z^{\nu}$, and, at high temperature and small activity (gaseous phase), we prove their existence, their analiticity in the interaction potentials and find a cluster property for them, generalizing to the case of many body potentials the results and the technics of references $[1,2,4]$ in the case of two body interactions (for further results in the two body case, see [3-6]). We also study the relation of our results with the work of RUELLE [7] about the ergodicity of the equilibrium states establishing that at any temperature and sufficiently small activity the equilibrium state is effectively a pure phase.

We assume that at each lattice point there can be either 0 or 1 particle and suppose that the particles interact through symmetric, translationally invariant, many body potentials $\Phi^{(k)}\left(x_{1}, \ldots, x_{k}\right)$, where $\Phi^{(k)}\left(x_{1}, \ldots, x_{k}\right), k=1,2, \ldots$ is a function defined only for different arguments. We shall regard these potentials as a function on the finite substets of $Z^{v}$ defined as $\Phi(X)=\Phi^{(k)}\left(x_{1}, \ldots, x_{k}\right)$ if $X=\left\{x_{1}, \ldots, x_{k}\right\}$ is a finite subset (configuration) of $Z^{\nu}$. We put $\Phi(\emptyset)=0$.

The potential energy of a finite configuration $X$ will then be given by

$$
U_{\Phi}(X)=\sum_{S \subset X} \Phi(S) .
$$

We shall consider only potentials $\Phi$ such that:

$$
\|\Phi\|=\sum_{O \in S}|\Phi(S)|<+\infty
$$


where $O$ is the origin of the lattice; we remark that the norm defined by (2) is different (in fact larger) than the norm introduced in [8].

It follows easily that, if $N(X)$ denotes the number of points of $X$, we have the following stability relation:

$$
\left|U_{\Phi}(X)\right| \leqq N(X)\|\Phi\| \text {. }
$$

The set $\mathscr{B}$ of interactions $\Phi$ satisfying (2) is a real Banach space in the norm defined by (2). It will be useful to consider also the complex Banach space $\mathscr{B}^{c}$ of the complex potentials $\Phi$ satisfying (2).

We call $\mathscr{B}^{\prime}$ the subspace of $\mathscr{B}^{c}$ defined as

$$
\mathscr{B}^{\prime}=\left\{\Phi \in \mathscr{B}: \Phi^{(1)}=0\right\},
$$

and if $\Phi \in \mathscr{B}^{c}$ we can write $\Phi=\left(\Phi^{(1)}, \Phi^{\prime}\right)$ with $\Phi^{(1)} \in C$ and $\Phi^{\prime} \in \mathscr{B}^{\prime}$. We observe that $-\Phi^{(1)}$ has the interpretation of chemical potential and, if $\beta=(k T)^{-1}, z=e^{-\beta \Phi^{(1)}}$ is the activity. We also remark that if $\Phi^{\prime} \in \mathscr{B}^{\prime}$ then

$$
\left|U_{\Phi^{\prime}}(X)\right| \leqq \frac{1}{2}\left\|\Phi^{\prime}\right\| N(X) .
$$

In what follows we shall put, for convenience, $\beta=1$.

The grand partition function for our system, when it is enclosed in a finite region $\Lambda \subset Z^{v}$ and its particles interact through a potential $\Phi=\left(\Phi^{(1)}, \Phi^{\prime}\right) \in \mathscr{B}^{c}$, is :

$$
Z_{\Lambda}(\Phi)=\sum_{Y \subset A} e^{-U_{\Phi}(Y)}
$$

and the finite volume correlation functions relative to $A$ are defined, in the case $Z_{\Lambda}(\Phi) \neq 0$ (in particular if $\Phi$ is real), as

$$
\begin{array}{llll}
\varrho_{\Phi \Lambda}(X)=Z_{\Lambda}(\Phi)^{-1} \sum_{Y \supset X ; Y \subset \Lambda} e^{-U_{\Phi}(Y)} & \text { if } & X \subset \Lambda \\
\varrho_{\Phi \Lambda}(X)=0 & \text { if } & X \subset \Lambda .
\end{array}
$$

It is also useful to introduce the "averaged" finite volume correlation functions :

$$
\bar{\varrho}_{\Phi \Lambda}(X)=\sum_{a \in Z^{\nu}} N(\Lambda)^{-1} \varrho_{\Phi \Lambda}(X+a) .
$$

We shall say that $\Lambda \rightarrow \infty$ if $\Lambda$ is a cube centered at the origin and the sides of $\Lambda$ tend to $\infty$.

\section{$\$ 2$. Definitions and Inequalities}

In this section we give some definitions and prove two auxiliary propositions of technical character which will be useful in the sequel.

In what follows we make the convention that a sum running over a void set of indices is zero.

Given a finite set $X \subset Z^{v}$, we call $X^{(1)}$ the set obtained by substraction from $X$ of one of its points $x_{1}$, for instance its first point in the lexicographic order. 
If $X \cap Y=\emptyset$ we define $\forall \Phi \in \mathscr{B} c$

$$
\begin{aligned}
& U_{\Phi}^{(1)}(X)=\sum_{x_{1} \in T \subset X} \Phi(T), \\
& W_{\Phi}(X, Y)=\sum_{x_{1} \in T \subset X} \Phi(T \cup Y),
\end{aligned}
$$

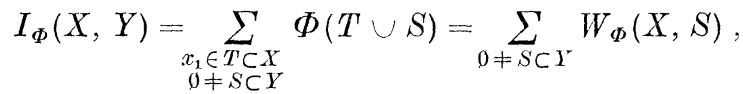

$$
\begin{aligned}
& K_{\Phi}(X, T)=\sum_{n \geqq 1} \sum_{\substack{\left\{S_{1}, \ldots, S_{n}\right\} \\
U_{i} S_{i}=T^{\prime}}} \prod_{j=1}^{r}\left(e^{-W_{\Phi}\left(X, S_{j}\right)}-1\right),
\end{aligned}
$$

the last summation runs over all $n \geqq 1$ and over all the subsets (containing $n$ elements) $\left\{S_{1} \ldots S_{n}\right\}$ of the set of non empty subsets of $T$ and such that $U_{i} S_{i}=T$. In what follows we shall always give this sense to the symbol $\sum_{n \geqq 1} \sum_{\substack{\left\{S_{1} \ldots S_{n}\right\} \\ U_{i} S_{i}=T}}$

We remark that $I_{\Phi}$ and $K_{\Phi}$ are independent of $\Phi^{(1)}$, i.e. $I_{\Phi}=I_{\Phi^{\prime}}$ and $K_{\Phi}=K_{\Phi^{\prime}}$. We have

$$
\begin{aligned}
& U_{\Phi}^{(1)}(X)=\Phi(1)+U_{\Phi^{\prime}}^{(1)}(X), \\
&\left|U_{\Phi^{\prime}}^{(1)}(X)\right| \leqq\left\|\Phi^{\prime}\right\|, \\
& \sum_{Y ; Y \cap X^{\prime}=0}\left|W_{\Phi}(X, Y)\right| \leqq\left\|\Phi^{\prime}\right\| \quad \text { at } X \text { fixed } \\
& e^{-I_{\Phi}(X, Y)}=\left(1+\sum_{T^{\prime} \subset Y} K_{\Phi}(X, T)\right) .
\end{aligned}
$$

We now prove

Proposition 1. Let $\Phi \in \mathscr{B}^{c}$, then for any $X$ fixed we have

$$
\sum_{T ; T^{\prime} \cap X=\emptyset}\left|K_{\Phi}(X, T)\right| \leqq \exp \left(e^{\mid \boldsymbol{\Phi}^{\prime \prime}}-1\right)-1 .
$$

In fact, since $\left|W_{\Phi}(X, S)\right| \leqq\left\|\Phi^{\prime}\right\|$ (see (15)) we have

$$
\left|e^{-W_{\Phi}\left(X, S^{\prime}\right)}-1\right| \leqq \frac{e^{\Phi^{\prime} \|}-1}{\left\|\Phi^{\prime}\right\|}\left|W_{\Phi}(X, S)\right|,
$$

then we can write the following chain of inequalities which proves (17): let $X$ be a fixed set:

$$
\begin{aligned}
& \sum_{T ; T \cap X=0}\left|K_{\Phi}(X, T)\right| \leqq \sum_{T ; T \cap X=0} \sum_{n \geqq 1} \sum_{\substack{\left\{S_{1} \ldots S_{n,}\right\} \\
U_{i} S_{i}=T^{\prime}}} \prod_{j=1}\left|e^{-W_{\Phi}\left(X, S_{j}\right)}-1\right| \\
& \leqq \sum_{T ; T \cap X=0} \sum_{n \geqq 1} \sum_{\substack{\left\{S_{1}, \ldots S_{n\}}\right\} \\
U_{i} S_{i}=T^{\prime}}}\left(\frac{e^{\left|\Phi^{\prime}\right|}-1}{\left\|\Phi^{\prime}\right\|}\right)^{n} \prod_{j=1}^{n}\left|W\left(X, S_{j}\right)\right|
\end{aligned}
$$

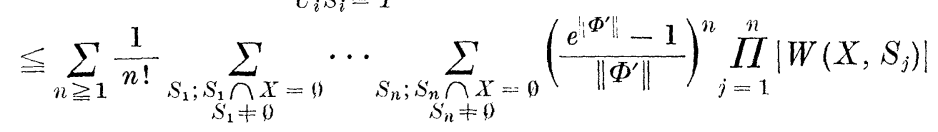


and using (15):

$$
\leqq \sum_{n \geqq 1} \frac{1}{n !}\left(e^{\mathscr{\Phi}^{\prime}}-1\right)^{n}=\exp \left(e^{\mid \Phi^{\prime}}-1\right)-1 .
$$

Now let $\lambda, \delta, \delta^{\prime}>0, \delta^{\prime}>\delta$ and let $S_{\varrho}(x)$ be the cube of side $\varrho$ centered at $x \in Z^{v}$. Define

$$
h_{\delta, \delta^{\prime}, \lambda}=\sup _{X \subset S \lambda(0)} \sum_{T ; T \cap X=0}\left|K_{\Phi}(X, T)\right|\left(\chi_{\lambda+\delta^{\prime}}(T)-\chi_{\lambda+\delta}(T)\right),
$$

where $\chi_{\varrho}(T)=1$ if $T \subset S_{\varrho}(0)$ and $\chi_{\varrho}(T)=0$ otherwise. Then

Proposition 2. The following limit exists:

$$
\lim _{\delta \rightarrow \infty} h_{\delta, \delta^{\prime}, \lambda}=0 \text {, }
$$

and holds uniformly in $\lambda$ and $0 \leqq \delta^{\prime}-\delta \leqq+\infty$.

In fact:

$$
h_{o, \delta^{\prime}, \lambda} \leqq \sup _{X \subset S \lambda(0)} \sum_{T ; T \cap X=0}\left|K_{\Phi}(X, T)\right|\left(1-\chi_{\lambda+\delta}(T)\right),
$$

and using (12) and (18) we get

$$
\begin{aligned}
& h_{\delta \delta^{\prime} \lambda} \leqq \sup _{X \subset S \lambda(0)} \sum_{T ; T \cap X=0} \sum_{n \geqq 1} \sum_{\substack{\left\{S_{1} \ldots . S_{n}\right\} \\
U_{i} S_{i}=T}} \prod_{j=1}^{n}\left|W_{\Phi}\left(X, S_{j}\right)\right|\left(\frac{e^{\Phi^{\prime}}-1}{\left\|\Phi^{\prime}\right\|}\right)^{n}
\end{aligned}
$$

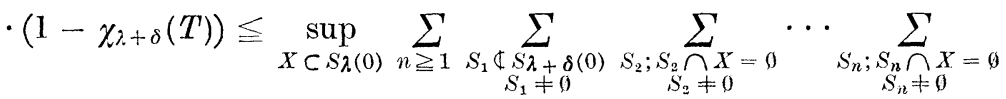

$$
\begin{aligned}
& \cdot \frac{1}{(n-1) !}\left(\frac{e^{\left\|\Phi^{\prime}\right\|}-1}{\left\|\Phi^{\prime}\right\|}\right)^{n} \prod_{j=1}^{n}\left|W_{\Phi}\left(X, S_{j}\right)\right| \leqq \sup _{X \subset S \lambda(0)} \sum_{S_{1} \varangle S_{\lambda+\delta}(0)} \\
& \cdot\left|W_{\Phi}\left(X, S_{1}\right)\right| \sum_{n \geqq 1} \frac{e^{\left\|\Phi^{\prime}\right\|}-1}{\left|\Phi^{\prime}\right|} \frac{\left(e^{\left\|\Phi^{\prime}\right\|}-1\right)^{n-1}}{(n-1) !} \leqq\left\|\Phi^{\prime}\right\|_{\delta} \\
& \cdot\left(\exp \left(e^{\mid \Phi^{\prime}}-1\right)\right) \frac{e^{\left\|\Phi^{\prime}\right\|}-1}{\left\|\Phi^{\prime}\right\|}
\end{aligned}
$$

where

$$
\left\|\Phi^{\prime}\right\|_{\delta}=\sum_{\substack{x_{1} \in Y \\ Y \nsubseteq S \delta\left(x_{1}\right)}}|\Phi(Y)|
$$

and, since $\left\|\Phi^{\prime}\right\|<+\infty$, we have $\lim _{\delta \rightarrow \infty}\left\|\Phi^{\prime}\right\|_{\delta}=0$, which gives the desired result.

\section{$\S$ 3. The Equations for the Correlation Functions}

From (9), (11) and (1) it follows that if $X \cap Y=\emptyset$

$$
U_{\Phi}(X \cup Y)=U_{\Phi}^{(1)}(X)+U_{\Phi}\left(X^{(1)} \cup Y\right)+I_{\Phi}(X, Y),
$$

hence using (17) and (16) we find, at $X \subset A$ fixed, and supposing 


$$
\begin{aligned}
& Z_{\Lambda}(\Phi) \neq 0 \text { : } \\
& \varrho_{\Phi}(X)=Z_{\Lambda}(\Phi)^{-1} \sum_{\substack{Y \\
Y \subset A}} e^{-U_{\Phi}^{(1)}(X)} e^{-U_{\Phi}\left(X^{(1)} \cup Y\right)} e^{-I_{\Phi}(X, Y)} \\
& =Z_{\Lambda}(\Phi)^{-1} \sum_{\substack{Y \subset X=0 \\
Y \subset A}} e^{-I_{\Phi}^{(1)}(X)} e^{-U_{\Phi}\left(X^{(1)} \cup Y\right)}\left(1+\sum_{T \subset Y} K_{\Phi}(X, T)\right) \\
& =e^{-U_{\Phi}^{(1)}(X)}\left(\varrho_{\Phi \Lambda}\left(X^{(1)}\right)-\varrho_{\Phi A}(X)+\sum_{R \cap X=\emptyset} K_{\Phi}(X, R)\right. \\
& \cdot\left(\varrho_{\Phi \Lambda}\left(X^{(1)} \cup R\right)-\varrho_{\Phi \Lambda}(X \cup R)\right) .
\end{aligned}
$$

Now we introduce the space $\mathscr{E}$ of complex functions $\varphi$ on the non empty finite subsets of $Z^{v}$ such that

$$
|\varphi|=\sup _{X}|\varphi(X)|<+\infty,
$$

in this norm $\mathscr{E}$ becomes a complex Banach space. Since, for $\Lambda$ finite, $\varrho_{\Phi \Lambda}(X)$, if defined, is different from zero only on a finite number of sets $X$ we can regard the function $X \rightarrow \varrho_{\Phi \Lambda}(X)$ as a vector $\varrho_{\Phi \Lambda} \in \mathscr{E}$ for all $\Phi \in \mathscr{B}^{c}$ such that $Z_{\Lambda}(\Phi) \neq 0$.

Let us define on $\mathscr{E}$ the operator $\mathscr{K}_{\Phi}$ as follows: let $\varphi \in \mathscr{E}$

$$
\begin{gathered}
\left(\mathscr{K}_{\Phi} \varphi\right)(X)=e^{-U_{\Phi}^{(1)}(x)}\left(-\varphi(X)+\sum_{S \cap X=\emptyset} K_{\Phi}(X, S)(\varphi(S)-\varphi(X \cup S))\right. \\
\left(\mathscr{K}_{\Phi} \varphi\right)(X)=e^{-U_{\Phi}^{(1)}(x)}\left(\varphi\left(X^{(1)}\right)-\varphi(X)+\sum_{S \cap X=\emptyset} K_{\Phi}(X, S)\right. \\
\cdot\left(\varphi\left(X^{(1)} \cup S\right)-\varphi(X \cup S)\right) \quad \text { if } N(X)>1
\end{gathered}
$$

then:

Proposition 3. $\forall \Phi \in \mathscr{B}^{c},(23)$ defines a continuous operator on $\mathscr{E}$.

In fact from (13), (17) and (22) we find, if $z=e^{-\Phi^{(1)}}$ :

$$
\left\|\mathscr{K}_{\Phi \|}\right\| 2|z| \exp \left(e^{\Phi^{\prime}}-1\right) .
$$

Let us define the operator $\chi_{A}$ on $\mathscr{E}$ as

$$
\left(\chi_{\Lambda} \varphi\right)(X)=\chi_{\Lambda}(X) p(X) \quad \varphi \in \mathscr{E}
$$

where $\chi_{\Lambda}(X)=0$ if $X \varangle \Lambda$ and $\chi_{\Lambda}(X)==1$ if $X \subset \Lambda$. We denote with $\alpha$ the vector $\alpha \in \mathscr{E}$ defined as

$$
\alpha(X)=1 \text { if } N(X)=1, \alpha(X)=0 \text { if } N(X)>1
$$

then the following theorem holds.

Theorem 1. Let $\Phi \in \mathscr{B}^{c}$, and let $\Lambda$ be a finite region:

i) If $Z_{\Lambda}(\Phi) \neq 0$ the correlation functions $\varrho_{\Phi A}$ verify the following equation

$$
\varrho_{\Phi \Lambda}=z \chi_{\Lambda} \alpha+\chi_{\Lambda} \mathscr{K}_{\Phi} \varrho_{\Phi A} .
$$

ii) If $\left\|\mathscr{K}_{\Phi}\right\|<1$ we have $Z_{\Lambda}(\Phi)+0$ and

$$
\begin{aligned}
\varrho_{\Phi \Lambda} & =z\left(1-\chi_{\Lambda} \mathscr{K}_{\Phi}\right)^{-1} \chi_{\Lambda} \alpha, \\
\left|\varrho_{\Phi \Lambda}\right| & \leqq|z|\left(1-\left\|\mathscr{K}_{\Phi}\right\|\right)^{-1} .
\end{aligned}
$$


iii) If $\left\|\mathscr{K}_{\Phi}\right\|<1$ then the equation

$$
\varrho_{\Phi}=z \alpha+\mathscr{K}_{\Phi} \varrho_{\Phi}
$$

has a unique solution $\varrho_{\Phi} \in \mathscr{E}$, this solution is translationally invariant and

$$
\lim _{\Lambda \rightarrow \infty} \varrho_{\Phi \Lambda}(X)=\varrho_{\Phi}(X) \quad \forall X \subset Z^{v}
$$

iv) If $\left\|\mathscr{K}_{\Phi}\right\|<1$ then we have also $\bar{\varrho}_{\Phi \Lambda} \in \mathscr{E}$ and

$$
\begin{gathered}
\left|\bar{\varrho}_{\Phi \Lambda}\right| \leqq|z|\left(1-\left\|\mathscr{K}_{\Phi}\right\|\right)^{-1} \cdot \forall A \subset Z^{v} \\
\lim _{\Lambda \rightarrow \infty} \bar{\varrho}_{\Phi \Lambda}(X)=\varrho_{\Phi}(X) .
\end{gathered}
$$

Remark. We observe that Eq. (27) do not reduce to the well known Kirkwood-Salzburg equations $[1,10]$ even in the case when only twobody interactions are present.

Part i) follows from $(2 \mathrm{I}),(23),(25),(26)$. To prove that $Z_{\Lambda}(\Phi) \neq 0$ if $\| \mathscr{K}_{\Phi \|}<1$ suppose $Z_{\Lambda}(\Phi)=0$, then if we consider the functions

$$
\begin{array}{ll}
R_{\Lambda \Phi}(X)=\sum_{Y \cap X=\emptyset ; Y \subset A} e^{-U_{\Phi}(X \cup Y)} & \text { if } \\
R_{\Lambda \Phi}(X)=0 & \text { if } X \subset \Lambda
\end{array}
$$

we find easily that the functions $R_{\Lambda \Phi} \in \mathscr{E}$ defined by $X \rightarrow R_{\Lambda \Phi}(X)$ verify the following equation:

$$
R_{\Lambda \Phi}=\chi_{\Lambda} \mathscr{K}_{\Phi} R_{\Lambda \Phi}
$$

and since $\left\|\mathscr{K}_{\Phi}\right\|<1$ this implies $R_{\Lambda \Phi}=0$, but $R_{\Lambda \Phi}(\Lambda)=e^{-U_{\Phi}(\Lambda)} \neq 0$; the other statements of part (ii) follows directly from (i). The first statement of iii) is clear; to prove (31) we use proposition 2 (formula (19)) and apply the same argument as ref. [1] (theorem 1, proof). The first statement of iv) follows from (29) and the definition (8) of $\bar{\varrho}_{\Phi_{\Lambda}}$; to prove (33) we note that because of the uniformity of (19) we may take $\lambda$ to be a function $\lambda(\delta)$ of $\delta$ and from the argument used in ref. [1] to prove (31) it follows that

$$
\left|\varrho_{\Phi \Lambda}(X)-\varrho_{\Phi}(X)\right|<\varepsilon \quad \forall X \subset S_{\lambda(\delta)}(0)
$$

if $A=S_{\lambda(\delta)+\delta}(0)$ and $\delta$ is sufficiently great. Now let $\Lambda \rightarrow \infty$ and choose $\lambda(\delta)=\delta^{2}$, then the volume of $S_{\lambda(\delta)+\delta}(0)$ is asymptotically equal, as $\Lambda \rightarrow \infty$, to the volume of $S_{\lambda(\delta)}(0)$. These facts imply that, fixed $X$, except for a surface effect in $a, \varrho_{\Phi}(X+a)$ will be equal within $\varepsilon$ to $\varrho_{\Phi}(X+a)$ $=\varrho_{\Phi}(X)$, while the importance of the surface effect in the expression of $\bar{\varrho}_{\Phi \Lambda}$ will result, as a consequence of $(29)$, in a term of the order of

$$
\left(V\left(S_{\lambda(\delta)+\delta}(0)\right)-V\left(S_{\lambda(\delta)}(0)\right) / V\left(S_{\lambda(\delta)}(0)\right) \underset{\Lambda \rightarrow \infty}{\longrightarrow} 0 .\right.
$$




\section{$\S 4$. Continuity and Analiticity Properties of $\mathscr{K}_{\Phi}$}

In this section we derive some auxiliary results on the analiticity of the dependence of the operator $\mathscr{K}_{\Phi}$ on $\Phi$. We shall use these results in the next section to derive the promised analiticity properties of pressure and correlation functions.

Proposition 4. Let $\Phi, \Psi \in \mathscr{B}^{c}$; then there exists a continuous function defined on all the two-dimensional plane $(x, y) \rightarrow f(x, y)$ such that

$$
\left\|\mathscr{K}_{\Phi}-\mathscr{K}_{\Psi}\right\| \leqq f(\|\Phi\|,\|\Psi\|)\|\Phi-\Psi\| .
$$

In fact from (23) we see that $\mathscr{K}_{\Phi}$ can be naturally decomposed in the product of a multiplication operator by $e^{-U_{\Phi}^{1}(X)}$ and an operator $\tilde{\mathscr{K}}_{\phi}$. The multiplication operator is norm continuous in $\Phi$ because of

$$
\sup _{X}\left|c^{-U_{\Phi}^{(1)}(X)}-e^{-U_{\Psi}^{(1)}(X)}\right| \leqq e^{\| \Phi}\left(e^{\mid \Phi-\Psi \|}-1\right) \leqq e^{\|\Phi\|} \frac{e|| \Phi\|+\| \Psi \|-1}{\|\Phi\|+\|\Psi\|}\|\Psi-\Phi\| .
$$

To show the continuity of $\mathscr{K}_{\Phi}$ in $\Phi$ we have thus only to show the continuity of $\tilde{\mathscr{K}}_{\Phi}$ : we have

$$
\begin{aligned}
& \left\|\tilde{\mathscr{K}}_{\Phi}-\tilde{\mathscr{K}}_{\Psi}\right\| \leqq \sup _{X} 2 \sum_{S \cap X=0}\left|K_{\Phi}(X, S)-K_{\Psi}(X, S)\right| \\
& \leqq 2 \sup _{X} \sum_{S \cap X=0} \sum_{n \geqq 1} \sum_{\substack{\left\{S_{1} \ldots S_{n}\right\} \\
U_{i} S_{i}=S}}\left|\prod_{j=1}\left(e^{-W_{\Phi}\left(X, S_{j}\right)}-1\right)-\prod_{j=1}\left(e^{-W_{\Psi}\left(X . S_{j}\right)}-1\right)\right| .
\end{aligned}
$$

Now we use the fact that if $f\left(z_{1}, \ldots, z_{n}\right)$ is an entire function of $z_{1}, \ldots, z_{n} \in C^{n}$ we have

$$
\left|f\left(z_{1}, \ldots, z_{n}\right)-f\left(z_{01}, \ldots, z_{0}\right)\right| \leqq\left|\sum_{i=1}^{n}\left(\operatorname{grad}_{i} f\right)\left(z_{i}-z_{0 i}\right)\right|
$$

where the gradient is evaluated at a suitable point of the segment joining $\left(z_{1}, \ldots, z_{n}\right)$ to $\left(z_{01}, \ldots, z_{0 n}\right)$ i.e. at a point of the form

$$
\left(\alpha z_{1}+(1-\alpha) z_{0 i}, \ldots, \alpha z_{n}+(1-\alpha) z_{0 n}\right) \quad 0 \leqq \alpha \leqq 1 ;
$$

applying (37) we get:

$$
\begin{aligned}
& \left\|\tilde{\mathscr{K}}_{\Phi}-\tilde{\mathscr{K}}_{\Psi}\right\| \\
& \leqq 2 \sup _{X} \sum_{S \cap X=0} \sum_{n \geqq 1} \sum_{\substack{\left\{S_{1} \ldots S_{n}\right\} \\
U_{i} S_{i}=S}} \sum_{k=1}^{n} \prod_{j \neq k}\left(e^{-\alpha W_{\Phi}\left(X, S_{j}\right)-(1-\alpha) W_{\Psi}\left(X, S_{j}\right)}-1\right) \\
& \cdot e^{-\alpha W_{\Phi}\left(X, S_{k}\right)+(1-\alpha) W_{\Psi}\left(X, S_{k}\right)}\left(W_{\Phi}\left(X, S_{k}\right)-W_{\Psi}\left(X, S_{k}\right)\right) \mid \text {, }
\end{aligned}
$$

where we have not explicitly written in the $\alpha$ 's the variables on which 
they depend; using (18) the chain of inequalities continues as

$$
\begin{aligned}
\leqq 2 \sup _{X} \sum_{S \cap X=0} \sum_{n \geqq 1} \sum_{\substack{\left\{S_{1} \ldots S_{n}\right\} \\
U_{i} S_{i}=S}} \sum_{k=1}^{n}\left[\left(\frac{e\left\|\Phi^{\prime}\right\|+\left\|\Psi^{\prime}\right\|-1}{\left\|\Phi^{\prime}\right\|+\left\|\Psi^{\prime}\right\|}\right)^{n-1}\right. \\
\left.\cdot e^{\left\|\Phi^{\prime}\right\|+\left\|\Psi^{\prime}\right\|} \prod_{j \neq k}\left(\left|W_{\Phi}\left(X, S_{j}\right)\right|+\mid W_{\Psi}\left(X, S_{j}\right)\right)\left|W_{\Phi-\Psi}\left(X, S_{k}\right)\right|\right]
\end{aligned}
$$

now proceeding as in the proof of proposition 1 we find that this last quantity is majorized by

$$
2 e^{\left\|\Phi^{\prime}\right\|+\left\|\Psi^{\prime}\right\|} \exp \left(e^{\left\|\Phi^{\prime}\right\|+\left\|\Psi^{\prime}\right\|}-1\right) \quad\|\Psi-\Phi\|,
$$

combining this result with (36) and (24) we get the result (35).

Now we investigate the analiticity of $\mathscr{K}_{\Phi+\lambda \Psi}$ in the complex variable $\lambda$. Let us define the kernel $\frac{\partial K_{\Phi}}{\partial \Psi}(X, T)$ as :

$$
\begin{aligned}
& \frac{\partial K_{\Phi}}{\partial \Psi}(X, T) \\
& \quad=-\sum_{n \geqq 1} \sum_{\substack{\left\{S_{1} \ldots S_{n\}}\right\} \\
U_{i} S_{i}=T}} \sum_{k=1}^{n} \prod_{j \neq k}\left(e^{-W_{\Phi}\left(X, S_{j}\right)}-1\right) e^{-W \Phi\left(X, S_{k}\right)} W_{\Psi}\left(X, S_{k}\right)
\end{aligned}
$$

and the operator $\frac{\partial \mathscr{K} \Phi}{\partial \Psi}$ on $\mathscr{E}$ as:

$$
\begin{aligned}
& \left(\frac{\partial \mathscr{K}_{\Phi}}{\partial \Psi} \varphi\right)(X)=-U_{\Psi}^{(1)}(X)\left(\mathscr{K}_{\Phi} \varphi\right)(X) \\
& \quad+e^{-U_{\Phi}^{(1)}(X)} \sum_{T \cap X=0} \frac{\partial K_{\Phi}}{\partial \Psi}(X, T)\left(\varphi\left(X^{(1)} \cup T\right)-\varphi(X \cup T)\right)
\end{aligned}
$$

then the following proposition holds:

Proposition 5. Let $\Phi, \Psi \in \mathscr{B}^{c}$ :

i) The operator $\frac{\partial \mathscr{K}_{\Phi}}{\partial \Psi}$ is a continuous operator on $\mathscr{E}$ and there exists a real continuous function $g:(x, y) \rightarrow g(x, y)$ defined on all the plane and such that

$$
\left\|\frac{\partial \mathscr{K}_{\Phi}}{\partial \Psi}\right\| \leqq g(\|\Phi\|,\|\Psi\|)
$$

ii) There exists a continuous function $l:(x, y) \rightarrow l(x, y)$ defined on all the plane and such that

$$
\left\|\frac{\partial \mathscr{K}_{\Phi}}{\partial \Psi}-\frac{\mathscr{K}_{\Phi+\lambda \Psi}-\mathscr{K}_{\Phi}}{\lambda}\right\| \leqq l(\|\Phi\|,\|\Psi\|)|\lambda|
$$

hence, in particular, $\mathscr{H}_{\Phi_{+} \Psi}$ is entire analytic in $\Psi$ in the sense that $\mathscr{K}_{\phi+\sum_{1}^{n} \lambda_{i} \Psi_{i}}$ is entire norm analytic in $\lambda_{1}, \ldots, \lambda_{n}[9]$.

The proof is obtained with the same technics as for propositions 1 and 4 . 


\section{§ 5. Pure Phases and Meyer's Expansion}

In order to prove the ergodicity of the equilibrium states when $\left\|\mathscr{K}_{\Phi}\right\|<1$ we report the following theorem:

Theorem 2. Let $\Phi, \Psi \in \mathscr{B}$.

i) The limit

$$
\lim _{\Lambda \rightarrow \infty} N(\Lambda)^{-1} \lg Z_{\Lambda}(\Phi)=P(\Phi)
$$

exists and defines a continuous convex function on $\mathscr{B}$.

ii) Let us define $\alpha_{\Phi A} \in \mathscr{B} *$ as:

$$
\alpha_{\Phi \Lambda}(\Psi)=\sum_{0 \in X} \Psi(X) \varrho_{\Phi \Lambda}(X) N(X)^{-1} \quad \Psi \in \mathscr{B},
$$

then if $\lim _{\Lambda \rightarrow \infty} \alpha_{\Lambda \Phi}(\Psi)$ exists for any $\Psi$ it defines a tangent plane to the graph of $P(\cdot)$ at $\Phi$ i.e. a linear functional $\alpha_{\Phi} \in \mathscr{B} *$ such that

$$
P(\Phi+\Psi) \geqq P(\Phi)-\alpha_{\Phi}(\Psi) \text {. }
$$

iii) If $\left.\frac{d P(\Phi+\lambda \Psi)}{d \lambda}\right|_{\lambda=0}$ exists for fixed $\Phi$ and $\Psi$, then for such $\Phi$ and $\Psi$ the limit $\lim _{\Lambda \rightarrow \infty} \alpha_{\Phi \Lambda}(\Psi)$ exists and

$$
\lim _{\Lambda \rightarrow \infty} \alpha_{\Phi \Lambda}(\Psi)=-\left.\frac{d P(\Phi+\lambda \Psi)}{d \lambda}\right|_{\lambda=0}
$$

iv) A necessary and sufficient condition for the existence of a unique tangent plane to the graph of $P(\cdot)$ at $\Phi$ is the existence for all $\Psi$ of $\left.\frac{d P(\Phi+\lambda \Psi)}{d \lambda}\right|_{\lambda=0}$.

This theorem can be proved as in ref. [8] (theorem 2 and remark): we have only to verify that the proof of the similar results of ref. [8] can be carried out, without any change, with the norm for the potentials introduced in (2).

Now we can state the following theorem:

Theorem 3. Let $\Phi \in \mathscr{B}^{c},\left\|\mathscr{K}_{\Phi}\right\|<1$; if $H_{\Phi} \subset \mathscr{B}^{c}$ denotes the largest open sphere, with radius $r_{\Phi}$, around the origin such that $\Psi \in H_{\Phi}$ implies $\left\|\mathscr{K}_{\Phi+\Psi}\right\|<1$, and if $\alpha_{\Phi} \in \mathscr{B}^{c *}$ is defined as

then

$$
\alpha_{\Phi}(\Psi)=\sum_{0 \in X} \Psi(X) \varrho_{\Phi}(X) N(X)^{-1} \quad \Psi \in \mathscr{B}^{c},
$$

i) the vectors $\varrho_{\Phi+\Psi}$ defined in (30) are norm analytic with respect to $\Psi \in H_{\Phi}$.

ii) Let $\Phi \in \mathscr{B}$ and $\Psi \in H_{\Phi}$, then if we define $\alpha_{\Phi \Lambda}(\Psi)$ as in (45) and $P_{A}(\cdot)$ as:

the following limit

$$
P_{\Lambda}(\Phi+\Psi)=N(\Lambda)^{-1} \lg Z_{\Lambda}(\Phi)-\int_{0}^{1} \alpha_{\Phi+\lambda \Psi, \Lambda}(\Psi) d \lambda
$$

$$
\lim _{\Lambda \rightarrow \infty} P_{\Lambda}(\Phi+\Psi)=P(\Phi+\Psi)
$$


exists, defines an analytic function of $\Psi \in H_{\Phi}$, and if $\Psi \in \mathscr{B} \cap H_{\Phi}$ coincides with the thermodynamic pressure defined by (44).

iii) If $\Phi \in \mathscr{B}, \varepsilon>0,\|\Psi\| \leqq r_{\Phi}-\varepsilon$ then the following formulae hold:

where

$$
\begin{gathered}
P(\Phi+\Psi)=P(\Phi)-\int_{0}^{1} \alpha_{\Phi+\lambda \Psi}(\Psi) d \lambda, \\
|P(\Phi+\Psi)| \leqq f(\Phi, \varepsilon)<+\infty
\end{gathered}
$$

$$
f(\Phi, \varepsilon)=P(\Phi)+\left(r_{\Phi}-\varepsilon\right) \sup _{\| \Psi^{\|} \leqq r_{\Phi}-\varepsilon}\left|e^{-\Phi(1)-\Psi^{(1)}}\left(1-\left\|\mathscr{K}_{\Phi+\Psi}\right\|\right)^{-1}\right| .
$$

iv) If we define, for $\Phi \in \mathscr{B}$ and $\Psi_{1}, \ldots, \Psi_{k} \in \mathscr{B}^{c}$ :

$$
A_{\Phi}^{(k)}\left(\Psi_{1}, \ldots, \Psi_{k}\right)=-\left.\frac{\partial^{k}}{\partial z_{1}, \ldots \partial z_{k}} P\left(\Phi+\sum_{i=1}^{k} z_{i} \Psi_{i}\right)\right|_{z_{1}=\cdots=z_{k}=0}
$$

then $A_{\Phi}^{(k)}(\cdot)$ is a k-linear symmetric continuous form on $\mathscr{B}^{c}$ and

$$
\left|A_{\Phi}^{(k)}\left(\Psi_{1}, \ldots, \Psi_{k}\right)\right| \leqq \frac{k^{k} f(\Phi, \varepsilon)}{\left(r_{\Phi}-\varepsilon\right)^{k}}\left\|\Psi_{1}\right\| \ldots\left\|\Psi_{k}\right\| .
$$

v) If we put $\alpha_{\Phi}^{(k)}(\Psi)=A_{\Phi}^{(k)}(\Psi, \ldots, \Psi)$, the following "Meyer" expansion holds for $\Psi \in H_{\Phi}$ :

$$
P(\Phi+\Psi)=P(\Phi)-\sum_{k=1}^{\infty} \frac{\alpha_{\phi}^{(h)}(\Psi)}{k !}
$$

and the series is absolutely convergent.

vi) If $\Phi \in \mathscr{B}$ we have

$$
\alpha_{\Phi}(\Psi)=\alpha_{\Phi}^{(1)}(\Psi)
$$

and the restriction to $\mathscr{B}$ of $\alpha_{\Phi} \in \mathscr{B}^{c *}$ defines a tangent plane to the graph of $P(\cdot)$ at $\Phi$ and this plane is the unique tangent plane at $\Phi$. Then the equilibrium state at the potential $\Phi$ is a pure thermodynamic phase (ergodic state) [7].

Part i) follows from the formula (remember that $z=e^{-\Phi_{(1)}}$ ):

$$
\varrho_{\Phi+\Psi}=e^{-\Phi(1)-\Psi^{(1)}}\left(1-\mathscr{K}_{\Phi+\Psi^{\prime}}\right)^{-1} \alpha,
$$

valid when $\left\|\mathscr{K}_{\Phi+\Psi}\right\|<1$ and from the norm analiticity of $\mathscr{K}_{\Phi+\Psi}$ in $\Psi$ (see Proposition 5).

To prove part ii) we observe that, as a consequence of (32), (33) we have

$$
\lim _{\Lambda \rightarrow \infty} \alpha_{\Phi \Lambda}(\Psi)=\alpha_{\Phi}(\Psi) \quad \forall \Psi \in \mathscr{B}^{c}
$$

furthermore as a consequence of i) $\alpha_{\Phi+\lambda \Psi}(\Psi)$ is continuous and as a consequence of $(32)$

$$
\begin{aligned}
\left|\alpha_{\Phi+\lambda \Psi, \Lambda}(\Psi)\right| & \leqq\left|e^{-\Phi^{(1)}-\lambda \Psi^{(1)}}\right|\left(1-\left\|\mathscr{K}_{\Phi+\lambda \Psi}\right\|\right)^{-1}\|\Psi\| \\
\left|\alpha_{\Phi+\lambda \Psi}(\Psi)\right| & \leqq\left|e^{-\Phi^{(1)}-\lambda \Psi^{(1)}}\right|\left(1-\left\|\mathscr{K}_{\Phi+\lambda \Psi}\right\|\right)^{-1}\|\Psi\|,
\end{aligned}
$$


then applying (44) and the dominated convergence theorem we get (50) and (51). From i) it follows that $\alpha_{\Phi+\lambda \Psi}(\Psi)$ is analytic in $\Psi \in H_{\Phi}$ and uniformly bounded when $\Psi$ varies in any closed sphere contained in $H_{\Phi}$ and $0 \leqq \lambda \leqq 1$, this implies the analiticity of $P(\Phi+\Psi)$ in $\Psi \in H_{\Phi}$.

The unproved part of iii) follows from (51) and (57). Part iv) can be proven as follows: let $z_{1}, \ldots, z_{k} \in C$ and

$$
\left|z_{i}\right|<\frac{r_{\Phi}-\varepsilon}{k\left\|\Psi_{i}\right\|} i=1,2, \ldots, k
$$

then $\sum_{i} z_{i} \Psi_{i} \in H_{\Phi}$ and we can write

$$
\begin{aligned}
& \left.\frac{\partial^{k} P\left(\Phi+\sum_{i=1}^{k} z_{i} \Psi_{i}\right)}{\partial z_{1} \ldots \partial z_{k}}\right|_{z_{1}=\cdots=z_{k}=0} \\
& =\frac{1}{(2 \pi i)^{k}} \oint \cdots \oint \frac{P\left(\Phi+\sum_{i=1}^{k} \lambda_{i} \Psi_{i}\right)}{\lambda_{1}^{2} \ldots \lambda_{k}^{2}} d \lambda_{1} \ldots d \lambda_{k}
\end{aligned}
$$

where the integrals are over circles of the $\lambda_{i}$-planes of radii $\frac{r_{\Phi}-\varepsilon}{k\left\|\Psi_{i}\right\|}$ and center at zero. Now (54) and the symmetry property follow from this integral expression and from (52). The linearity property follows from the analiticity in $z_{1}, \ldots, z_{k}$ of $P\left(\Phi+\sum_{i}^{k} z_{i} \Psi_{i}\right)$ around zero and from the formula (obtained from (51)):

$$
\left.\frac{\partial P\left(\Phi+\sum_{i=1}^{k-1} z_{i} \Psi_{i}+z_{k} \Psi_{k}\right)}{\partial z_{k}}\right|_{z_{k}=0}=-\alpha_{\Phi+\sum_{i=1}^{k} \sum_{i}^{1} z_{i} \Psi_{i}}\left(\Psi_{k_{k}}\right),
$$

which shows that $\left.\frac{\partial P\left(\Phi+\sum_{i}^{k-1} z_{i} \Psi_{i}+z_{k} \Psi_{k}\right)}{\partial z_{k}}\right|_{z_{k}=0}$ is linear in $\Psi_{k}$. If we set in this last formula $z_{1}=z_{2}=\cdots=z_{k-1}=0$ we prove (56); the remaining part of iv) follows from part iv) of theorem 2 and from the fact that, knowing that at $\Phi$ there is a unique tangent plane to the graph of $P(\cdot)$, we can apply the results of ref. [7] (theorem 4, part iii)) to guarantee that at $\Phi$, the equilibrium state of the system is a pure thermodynamic phase: we have only to remark that the potentials of $\mathscr{B}$ satisfy also condition (1.2) of the paper referred to.

Remark. We obtain a physical interpretation of the results of theorem 3 reintroducing the inverse temperature $\beta$ and observing from (24) that, for fixed $\beta$, if the activity $z$ is small, we have $\left\|\mathscr{K}_{\beta \Phi}\right\|<1$. 


\section{\$ 6. Cluster Properties of Correlation Functions}

Suppose that the graph of $P(\cdot)$ has a unique tangent plane corresponding to $\Phi \in \mathscr{B}$, then the infinite volume correlation functions $\varrho_{\Phi}$ are uniquely determined and define a tangent functional to the graph of $P(\cdot)$.

We can then define the cluster (truncated) functions $\varrho_{\Phi}^{T}$ by means of the relations (where $7(X)=0$ if $X \neq \emptyset, 1(\emptyset)=1$ ):

$$
\begin{aligned}
& \varrho_{\Phi}^{T}(\emptyset)=0 \\
& \varrho_{\Phi}(X)=1(X)+\sum_{n \geqq 1} \sum_{\substack{\left.X_{1}, \ldots X_{n}\right\} \\
U_{i} X_{i}=X \\
\Sigma_{i} N\left(X_{i}\right)=N(X)}} \prod_{i=1}^{n} \varrho_{\Phi}^{T}\left(X_{i}\right)
\end{aligned}
$$

(for the meaning of the summation symbols see section 2).

In this section we shall prove that at fixed temperature and sufficiently small activity the following cluster property holds:

$$
\sum_{\substack{0 \in X \\ N(X) \text { fixed }}}\left|\varrho_{\Phi}^{T}(X)\right|<+\infty .
$$

This result will be a consequence of theorem 4 which will be proved with the same technics as in reference [4]. In order to formulate this theorem we consider the finite configurations $X$ which we allow to possess multiplicities. If $X$ is such a configuration we denote $\tilde{X}$ the set of points occupied by the particles of $X$ : hence a configuration $X$ is determined by giving $\widetilde{X}$ and the multiplicity of every point of $\tilde{X}$. We denote $N(X)$ the number of particles of the configuration $X$. The reason for the introduction of these "unphysical" configurations will become clear when we shall introduce the operator $D_{x}$.

Let $K$ be the space of all the configurations just defined, and let $\mathscr{F}$ be the space of complex functions $f$ on $K$ such that for each $n$

$$
\sup _{N(X)=n}|f(X)|=|f|_{n}<+\infty .
$$

Now we define some operations on or between elements of $\mathscr{F}$. Let $\varphi_{1}, \varphi_{2} \in \mathscr{F}$, we define the product $\varphi_{1} \cdot \varphi_{2} \in \mathscr{F}$ as

$$
\left(\varphi_{1} \cdot \varphi_{2}\right)(X)=\sum_{Y \subset X} \varphi_{1}(Y) \varphi_{2}(X / Y)=\left(\varphi_{2} \cdot \varphi_{1}\right)(X)
$$

where $X / Y$ is the configuration obtained from $X$ by substracting the points of $Y$ (taking multiplicity into account).

Let $\varphi \in \mathscr{F}_{0}$ where $\mathscr{F}_{0}=\{\varphi \in \mathscr{F}: \varphi(\emptyset)=0\}$, then we define the exponential $\Gamma \varphi$ of $\varphi$ as a mapping between $\mathscr{F}_{0}$ and $\mathscr{F}_{1}$ where $\mathscr{F}_{1}$ $=\{\varphi \in \mathscr{F}: \varphi(\emptyset)=1\}$ defined, putting $\varphi^{0}(X)=1(X)$, as:

$$
(\Gamma \varphi)(X)=\sum_{n \geqq 0} \frac{\varphi^{n}(X)}{n !}=1(X)+\sum_{\substack{\left.n \geqq 1 \\ n \\ \Sigma_{i} X_{1} \ldots X_{i}=X\right\} \\ N\left(X_{i}\right)=N(X)}} \prod_{i=1}^{n} \varphi\left(X_{i}\right) .
$$


It is easily verified that this mapping is defined on all of $\mathscr{F}_{0}$ and is one-to-one and onto $\mathscr{F}_{1}$.

Now we define a scalar product between elements of $\mathscr{F}$ and certain functions $\chi_{\Lambda}$ defined for regions $\Lambda \subset Z^{v}$ (not necessarily finite) as $\chi_{\Lambda}(X)=1$ if $X \subset \Lambda$ and $\chi_{\Lambda}(X)=0$ if $X \subset A:$ let $\mathscr{D}_{\Lambda}=\left\{\varphi \in \mathscr{F}: \sum_{\tilde{X} \subset A}|\varphi(X)|<+\infty\right\}$, then if $\varphi \in \mathscr{D}_{\Lambda}$ we define:

$$
\left\langle\chi_{11}, \varphi\right\rangle=\sum_{\tilde{X} \subset A} \varphi(X)=\sum_{X} \chi_{\Lambda}(X) \varphi(X) .
$$

If $\varphi_{1}, \varphi_{2} \in \mathscr{D}_{A}$ then $\varphi_{1} \cdot \varphi_{2} \in \mathscr{D}_{\Lambda}$ and

$$
\left\langle\chi_{\Lambda}, \varphi_{1} \cdot \varphi_{2}\right\rangle=\left\langle\chi_{\Lambda}, \varphi_{1}\right\rangle\left\langle\chi_{\Lambda}, \varphi_{2}\right\rangle \text {. }
$$

Finally we define a mapping $D_{X} \mathscr{F} \rightarrow \mathscr{F}$ as :

$$
\left(D_{X} \varphi\right)(Y)=\varphi(X \cup Y)
$$

where $X \cup Y$ denotes, here as well as in the remaining of this section, the configuration obtained from $Y$ by adjoining to it the configuration $X$ taking into account the multiplicities.

We find the following relations

$$
\begin{aligned}
& D_{X}\left(\varphi_{1} \cdot \varphi_{2}\right)=\varphi_{1} \cdot D_{X} \varphi_{2}+\varphi_{2} \cdot D_{X} \varphi_{1} \quad \text { if } N(X)=1, \\
& D_{X} \Gamma \varphi=\left(D_{X} \varphi\right)(\Gamma \varphi) \text { if } N(X)=1, \quad \forall \varphi \in \mathscr{F}_{0}, \\
& D_{X} \Gamma \varphi=\left(\sum_{\substack{\left.n \geqq 1 \\
\sum_{i} X_{i} X_{i} X_{n\}}\right\} \\
\sum_{i}\left(X_{i}\right)=N(X)}} \prod_{i=1}^{n}\left(D_{X_{i}} \varphi\right)\right) \cdot \Gamma \varphi \text { if } X \neq \emptyset, \quad \forall \varphi \in \mathscr{F}_{0} .
\end{aligned}
$$

Now to formulate the main theorem of this section we need only the expression of the cluster functions relative to finite regions $\Lambda$. Let $\Phi \in \mathscr{B}$ be such that the graph of $P(\cdot)$ admits a unique tangent plane at $\Phi$. Then let $\varrho_{\Phi}$ be the correlation functions, since $0 \leqq \varrho_{\Phi}(X) \leqq 1$ and $\varrho_{\Phi}(\emptyset)=1$, we can regard $\varrho_{\Phi}$ as an element of $\mathscr{F}_{1}$ if we put $\varrho_{\Phi}(X)=0$ when $X \neq \tilde{X}$. We define the truncated functions as an element of $\mathscr{F}_{0}$ through the relation:

$$
\varrho_{\Phi}=\Gamma \varrho_{\Phi}^{T} .
$$

Similar definitions are set for the finite volume correlation functions $\varrho_{\Phi \Lambda}$ (in particular $\varrho_{\Phi \Lambda}=\Gamma \varrho_{\Phi \Lambda}^{T}$ ).

Now let $\varphi_{\Phi} \in \mathscr{F}_{1}$ be defined as :

$$
\varphi_{\Phi}(X)=e^{-U_{\Phi}(X)} \text { if } X=\tilde{X} \text { and } \varphi_{\Phi}(X)=0 \text { if } X \neq \tilde{X} .
$$

It is easy to verify that, if $\varphi_{\Phi}^{-1} \cdot D_{X} \varphi_{\Phi} \in \mathscr{D}_{\Lambda}$, then:

$$
\varrho_{\Phi \Lambda}(X)=\frac{\left\langle\chi_{\Lambda}, D_{X} \varphi_{\Phi}\right\rangle}{\left\langle\chi_{\Lambda}, \varphi_{\Phi}\right\rangle}=\left\langle\chi_{\Lambda}, \varphi_{\Phi}^{-1} D_{X} \varphi_{\Phi}\right\rangle .
$$

If we define $\varphi_{\Phi}^{T} \in \mathscr{F}_{0}$ such that $\varphi_{\Phi}=\Gamma \varphi_{\Phi}^{T}$ and if we suppose that 
$D_{X} \varphi_{\Phi}^{T} \in \mathscr{D}_{\Lambda} \forall X, N(X) \geqq 1$ we find, using (71), (74)

$$
\varrho_{\Phi \Lambda}(X)=1(X)+\sum_{n \geqq 1} \sum_{\begin{array}{c}
\left\{X_{1} \ldots X_{n\}}\right\} \\
U_{i} X_{i}=X \\
\sum_{i} N\left(X_{i}\right)=N(X)
\end{array}}\left\langle\chi_{\Lambda}, D_{X_{i}} \varphi_{\Phi}^{T^{\prime}}\right\rangle,
$$

which shows (always if $\varphi_{\Phi}^{-1} \cdot D_{X} \varphi_{\Phi} \in \mathscr{D}_{\Lambda}$ and $D_{X} \varphi_{\Phi}^{T} \in \mathscr{D}_{A} \forall X, N(X) \geqq 1$ )

$$
\varrho_{\Phi \Lambda}^{T}(X)=\left\langle\chi_{\Lambda}, D_{X} \varphi_{\Phi}^{T}\right\rangle \text { if } N(X) \geqq 1
$$

Now the following theorem holds:

Theorem 4. If $\Phi \in \mathscr{B}$ and $c(\Phi)=2 z e^{\left\|\Phi^{\prime}\right\|} \exp \left(e^{\left\|\Phi^{\prime}\right\|}-1\right)<1$ we have

i) $\sum_{Y ; N(Y)=m}\left|\varphi_{\Phi}^{-1} D_{X} \varphi_{\Phi}(Y)\right| \leqq z c(\Phi)^{m+n-1}$ if $N(X)=n \geqq 1$.

ii) $D_{X} \varphi_{\Phi}^{T} \in \mathscr{D}_{Z^{v}} \quad \forall X, N(X) \geqq 1$.

iii) $\varrho_{\Phi}^{T}$, which is uniquely defined as a consequence of (24) and theorem 1 , is given by the expresssion:

$$
\varrho_{\Phi}^{T}(X)=\left\langle\chi_{Z^{v}}, D_{X} \varphi_{\Phi}^{T}\right\rangle \quad \forall X, N(X) \geqq 1 .
$$

iv) The following cluster property is valid:

$$
\sum_{\substack{O \in X \\ N(X)=m}}\left|\varrho_{\Phi}^{T}(X)\right| \leqq z \frac{c(\Phi)^{m_{2}-1}}{(1-c(\Phi))^{m}} .
$$

Remark. (79) is stronger than the promised (62) since in (79) we consider also configurations with multiplicity.

To prove this theorem we remark that iii) follows from ii) and i), and ii) will be proved if we prove it for $N(X)=1$ and in this case ii) follows from (70) and i). Hence we have only to prove i) and iv). Let us prove i): taking into account the explicit form (73) of $\varphi_{\Phi}$ and using the same technics as in the preceding sections we can prove the following equations which we write using the notations of sections 1,2 : if $N(X)$ $\geqq 1, N(Y) \geqq 0$ and, if we put $\Delta_{X}(Y)=\left(\varphi_{\Phi}^{-1} \cdot D_{X} \varphi_{\Phi}\right)(Y), Y_{1}=Y /\left\{x_{1}\right\}$, $\Theta_{x_{1}}(Y)=1$ if $x_{1} \in Y$ and $\Theta_{x_{1}}(Y)=0$ if $x_{1} \notin Y$, we get

$$
\begin{aligned}
\Delta_{X}(Y) & =e^{-U_{\Phi}^{(1)}(X)}\left[\Delta_{X^{(1)}}(Y)-\Theta_{x_{1}}(Y) \Delta_{X}\left(Y_{1}\right)\right. \\
& \left.+\sum_{T T^{\prime} \subset Y} K_{\Phi}(X, T)\left(\Delta_{X^{(1)} \cup T^{\prime}}(Y / T)-\Delta_{X \cup T^{\prime}}\left(Y_{1} / T\right) \Theta_{x_{1}}(Y)\right)\right]
\end{aligned}
$$

where $K_{\Phi}(X, T)$ and $e^{-U_{\Phi}^{(1)}(X)}$ are to be taken equal to zero if $T \neq \widetilde{T}$ or $X \neq \tilde{X}$.

Now we define

$$
I_{m}=\sup _{\substack{N(X)+N(Y)=m \\ N(X) \geqq 1, N(Y) \geqq 0}} \sum_{Y} \quad\left|\Delta_{X}(Y)\right| .
$$

20 Commun. math. Phys., Yol. 7 
We find using (17), (79) and the fact that $\Delta_{0}(Y)=1(Y)$ :

$$
I_{m} \leqq z e^{\left\|\Phi^{\prime}\right\|}\left[2 I_{m-1}+\left(\exp \left(e^{\left\|\Phi^{\prime}\right\|}-1\right)-1\right) 2 I_{m-1}\right] \quad m \geqq 2
$$

which, taking into account that $I_{1}=z$ gives

$$
I_{m} \leqq z c(\Phi)^{m-1} .
$$

To prove iv) we proceed as follows:

$$
\begin{aligned}
\sum_{\substack{0 \subset X \\
N(X)=m}}\left|\varrho_{\Phi}^{T}(X)\right|= & \sum_{T}\left|\sum_{n \geqq 0} \sum_{Y ; N(Y)=n} \varphi_{\Phi}^{T}(\{0\} \cup T \cup Y)\right| \\
& \leqq \sum_{n \geqq 0} \sum_{T ; N(T)=m-1} \sum_{Y ; N(Y)=n}\left|\varphi_{\Phi}^{T}(\{0\} \cup T \cup Y)\right| \\
& \leqq \sum_{n \geqq 0} S ; N(S)=m+n-1 \\
& \leqq \sum_{n \geqq 0}\left(\begin{array}{c}
m+n-1 \\
m-1
\end{array}\right) z c(\Phi)^{m+n-1}=z \frac{c(\Phi)^{m-1}}{(1-c(\Phi))^{m}}
\end{aligned}
$$

where we have used the formula $D_{\{0\}} \varphi_{\Phi}^{T}=\varphi_{\Phi}^{-1} \cdot D_{\{0\}} \varphi_{\Phi}$ and (77).

Acknowledgements. We are greatly indebted to D. RuELLE for having proposed the problem and suggested improvements. We also wish to thank J. GinIBRE and D. RUELLE for reading the manuscript and M. L. Motchane for his kind hospitality at the IHES. We are indebted to CNRS for financial support.

\section{References}

1. Ruelle, D.: Correlation functions of classical gases: Ann. Phys. (N. Y.) 25, 109 (1963).

2. Prnrose, O.: Convergence of fugacity expansions for fluids and lattice gases. J. Math. Phys. 4, 1312 (1963).

3. Lebowitz, J. L., and O. Penrose: Convergence of virial expansions . J. Math. Phys. 5, 841 (1964).

4. Ruelle, D.: Cluster property of correlation functions of classical gases. Rev. Mod. Phys. 36, 580 (1964).

5. Ginibre, J.: Reduced density matrices of quantum gases I. Limit of infinite volume. J. Math. Phys. 6, 238 (1965).

6. - Reduced density matrices of quantum gases II. Cluster property. J. Math. Phys. 6, 252 (1965).

7. Ruelte, D.: A variational formulation of equilibrium statistical mechanics. Commun. Math. Phys. 5, 321 (1967).

8. Gallavotti, G., and S. Mrracle-Sole: Statistical mechanics of lattice systems. Commun. Math. Phys. 5, 317 (1967).

9. Dunford, N., and J. Schwartz: Linear operators, vol. I. III.14 and VI.10.5. New York: Interscience 1958.

10. HrLL, T. L.: Statistical mechanics. New York: McGraw-Hill 1956.

Dr. G. Galla vottit

Dr. S. Miracle-Sole

Institut des Hautes Etudes Scientifiques

F 91 Bures-sur-Yvette 